\title{
VI. THE SYMPOSIA OF THE INTERNATIONAL ASTRONOMICAL UNION
}

There were 83 participants when the first General Assembly of the Union convened. By today's standards it was a medium-sized Symposium. It was not difficult to meet fellow participants and there was ample time and opportunity to discuss the interesting problems of that day. In the few decades following the first General Assembly astronomy developed rapidly; in particular, coverage became enormously broad. There is not a person today who could boast of keeping abreast of all new developments in astronomy. The membership of the Union grew accordingly and there remained few problems which were in the centre of interest of all I 600 Members of the IAU.

Parkinson would probably establish an exact formula for the number of participants of scientific meetings when the effectiveness is lost. We shall be satisfied with a rough estimate that an attendance of the order of 100 people at a meeting still allows a fruitful and lively discussion; each participant has the opportunity to give full vent to his ideas.

The increase in membership and coverage could have been faced in two ways. The first would have been to split the Union into several associations, each covering a branch of astronomy. Although this solution may prove to be inavoidable in the future, there are strong reasons for keeping all of astronomy together at present. Astronomy is one of the international sciences par excellence and there is still a single trend in the evolution of our science in the world. Each serious paper is based on results reached at all observatories, irrespective of country or continent. It would be difficult, if not impossible, to keep up this thoroughly international feature for a number of mutually almost independent associations.

The other possibility would have been to create smaller meetings with a strictly limited attendance and concerned with particular problems. This was the idea which led to the organization of Symposia, and it was a successful one. Symposia are organized according to the needs of any branch of astronomy and only experts united by a deep interest in the problems participate.

Besides the main goals, i.e. concentration of interest and the preservation of the unity of Astronomy, the Symposia offer several other advantages.

Experts in the different branches are brought together; e.g. at Symposium no. 20 "The Galaxy and the Magellanic Clouds", radioastronomers and optical astronomers met and discussed common problems approached by different methods and from different angles. At Symposium no. 25 "The Theory of Orbits in the Solar System and in Stellar Systems", stellar dynamists and celestial mechanists found that the three-body problem and motions of stars in the Galaxy pose the same questions and that the solutions can be expressed in analogical terms. Each Symposium gives an example of this kind.

Generally, the reactions of the scientific public to any result presented in a journal, are scattered in a number of other papers and appear much later. At a Symposium, the discussion follows immediately after the presentation of the results and the reader of the proceedings can easily compare the different opinions expressed.

The Symposia are thoroughly international and the Union usually makes a financial provision to this end. The importance of the international character of Symposia cannot be overstressed, as well from the standpoint of the development of science as from the standpoint of general understanding between people of different nations. 
The history of the Symposia of the Union is not a very long one (see above pp. 9 and 43 ). Prior to I953 some symposia were held during General Assemblies and are published in the Transactions. In 1953 a firmer basis of organization was established and a uniform series of Symposia created. Table I lists all Symposia of the new series. The title, date, place, joint organization with other scientific Unions, the editor and publishing house are shown. The longest monothematic series is on Cosmical Gas Dynamics (Symposia nos. 2, 6, 8, 12 and 28). Galactic structure was discussed at Symposia nos. I, 5, 7 and 20. There is, however, no clear-cut limit between the branches covered by individual Symposia because border-line problems are frequently dealt with.

The results arrived at by a small group of participants must be disseminated to the general scientific public. Thus the publishing of the proceedings of a Symposium is of vital importance. According to the possibilities existing in each case, proceedings have been published in scientific journals, as commercial undertakings by professional publishers, or by the Union. This diversity had the inconvenient consequence of losing the uniform character of a series. To prevent this, Symposia from no. 24 onwards, will be published by the Union.

The different aspects of IAU Symposia are discussed in more detail in Part 2 of this Handbook, page 129.

Details regarding the availability of published proceedings of Symposia are included in the list of IAU publications on pages 43 .

Table I

No.

Date
and
place

I. Co-ordination of Galactic Research

2. Gas Dynamics of Cosmic Clouds

3. Non-Stable Stars

4. Radio Astronomy

5. Comparison of Large-Scale Structure of the Galactic System with that of other Stellar Systems

6. Electromagnetic Phenomena August 1956 in Cosmical Physics

Stockholm

7. Second Conference on Coordination of Galactic Research

8. Third Symposium on Cosmical Gas Dynamics

June 1957

Saltsjöbaden

June 1957

Cambridge, Mass., U.S.A.,

9. Paris Symposium on Radio Astronomy

August ${ }_{1958}$

Paris

10. The Hertzsprung-Russell Diagram

August I958

Moscow

II. The Rotation of the Earth and Atomic Time Standards Moscow
Editor
and
Publishing house

Organized in co-operation with

\section{A. Blaauw}

Cambridge University Press, I955

H. C. van de Hulst and

J. M. Burgers

North-Holland Publ. Co., 1955

G. H. Herbig

Cambridge University Press, r957

H. C. van de Hulst

Cambridge University Press, 1957

N. G. Roman

Cambridge University Press, I958

IUTAM

B. Lehnert

IUPAP, Cambridge University Press, $195^{8}$ IUGG

A. Blaauw et al.

Cambridge University Press, 1959

J. M. Burgers and

R. N. Thomas

Reviews of Modern Physics, 1958

R. N. Bracewell

Stanford University Press, 1959

J. L. Greenstein

Annales d'Astrophysique, 1959

D. Brouwer

The Astronomical fournal, 1959
IUTAM

URSI 
No. Title
I2. Aerodynamic Phenomena
in Stellar Atmospheres
(Fourth Symposium on
Cosmical Gas Dynamics)

I3. The Future of the International Latitude Service

I4. The Moon

July I960
Helsinki
December 1960
Leningrad

August I96I

Santa Barbara

August I96r

Cloudcroft,

New Mexico
I7. Visual Double Stars

I8. Theoretical Interpretation of Upper Atmosphere Emissions

I9. Site Testing

20. The Galaxy and the Magellanic Clouds

\section{August 196r}

Berkeley

June 1962

Paris

October 1962

Rome

March 1963

Canberra and

Sydney

21. The System of Astronomical May 1963 Constants

22. Stellar and Solar Magnetic Fields

23. Astronomical Observations from Space Vehicles

24. Spectral Classification and Multicolour Photometry
Paris

September 1963

Munich

August 1964

Liège

August 1964

Saltsjöbaden
25. The Theory of Orbits in the August 1964 Solar System and in Stellar Thessaloniki Systems

26. Abundance Determination in Stellar Spectra

August 1964 Utrecht

P. Melchior

Bulletin Géodésique, $196 \mathbf{1}$

Editor
and

Publishing house

R. N. Thomas et al.

Il Nuovo Cimento, 1961

Z. Kopal and

Z. K. Mikhailov

Academic Press, I 962

G. C. McVittie

Macmillan Co., I962

J. W. Evans

Academic Press, 1963

S. L. Lippincott

Publications of the Astronomical

Society of the Pacific, 1962

D. R. Bates

Planetary and Space Science, 1963

J. Rösch et al.

Bulletin Astronomique, 1963

F. J. Kerr and

A. W. Rodgers

Australian Academy of Sciences, I964

J. Kovalevsky

Bulletin Astronomique, ${ }_{196} 6_{5}$

R. Lüst

North-Holland Publ. Co., I 965

J.-L. Steinberg

Annales d'Astrophysique, I964, I 965

K. Lodén, L. O. Lodén and

U. Sinnerstad

IAU, I966

G. Contopoulos

IAU, 1966

H. Hubenet

IAU, 1966

April 1965

Tucson, Arizona

D. L. Crawford

and Pasadena,

California

28. Cosmical Gas Dynamics (Fifth Symposium)
September 1965

Nice
R. N. Thomas

IAU
Organized in co-operation with

IU'TAM

IAG

IUGG

URSI

COSPAR

COSPAR

COSPAR

IUTAM 